\title{
Massive gravity: exorcising the ghost
}

\author{
Lasma Alberte, ${ }^{a}$ Ali $\mathbf{H}$. Chamseddine ${ }^{b, c}$ and Viatcheslav Mukhanov ${ }^{a, d}$ \\ a Theoretical Physics, Ludwig Maxmillians University, \\ Theresienstr. 37, 80333 Munich, Germany \\ ${ }^{b}$ American University of Beirut, Physics Department, \\ Beirut, Lebanon \\ ${ }^{c}$ I.H.E.S., \\ F-91440 Bures-sur-Yvette, France \\ ${ }^{d}$ Department of Physics, New York University, \\ New York, NY 10003, U.S.A. \\ E-mail: lasma.alberte@physik.uni-muenchen.de, chams@aub.edu.lb, \\ Viatcheslav.Mukhanov@physik. uni-muenchen.de
}

AbSTRACT: We consider Higgs massive gravity $[1,2]$ and investigate whether a nonlinear ghost in this theory can be avoided. We show that although the theory considered in $[10,11]$ is ghost free in the decoupling limit, the ghost nevertheless reappears in the fourth order away from the decoupling limit. We also demonstate that there is no direct relation between the value of the Vainshtein scale and the existence of nonlinear ghost. We discuss how massive gravity should be modified to avoid the appearence of the ghost.

KEywords: Classical Theories of Gravity, Spontaneous Symmetry Breaking, Gauge Symmetry

ArXIV EPRINT: 1011.0183v1 


\section{Contents}

$\begin{array}{llr}1 & \text { Introduction } & 1\end{array}$

2 Higgs massive gravity 2

3 Boulware-Deser nonlinear ghost $\quad 3$

4 Ghost in nonlinear extensions of massive gravity 5

$\begin{array}{lll}5 & \text { Can we avoid the nonlinear ghost? } & 7\end{array}$

6 How dangerous are ghosts? 10

$\begin{array}{lll}7 & \text { Conclusions } & 11\end{array}$

\section{Introduction}

In $[1,2]$ we have devised a Higgs mechanism for massive gravity and demonstrated how this theory goes smoothly to General Relativity below the Vainshtein radius [3], thus resolving the problem of van Dam, Veltman and Zakharov discontinuity $[4,5]$. This result, obtained in Higgs massive gravity, is in agreement with the results derived in bigravity theories in [6-8]. Moreover, we have found that the corresponding Vainshtein scale depends on the nonlinear extension of the Fierz-Pauli term [9]. In particular, it was shown that the Vainshtein scale can be changed within the range $M_{0}^{1 / 3} m_{g}^{-2 / 3}<R_{V}<M_{0}^{1 / 5} m_{g}^{-4 / 5}$, where $M_{0}$ and $m_{g}$ are, respectively, the mass of the external source and the mass of the graviton in Planck units. The class of actions which lead to different Vainshtein scales $R_{V}$ coincide with the actions derived in $[10,11]$. These were obtained from the requirement of absence of the nonlinear ghost [12] in the corresponding order of perturbation theory, in the decoupling limit when both the graviton mass and the gravitational constant simultaneously vanish in such a way that the appropriate Vainshtein scale is kept fixed. Moreover, there is a unique action (up to total derivatives), corresponding to $R_{V}^{\infty}=M_{0}^{1 / 3} m_{g}^{-2 / 3}$, in the decoupling limit, for which the Boulware-Deser ghost does not appear at all below Vainshtein energy scale up to an arbitrary order in perturbation theory [10, 11]. Therefore, a natural interesting question arises as to whether this result could be sustained if we consider instead of the decoupling limit (which is not physical), the full nonlinear theory of massive gravity. The answer to this question will also help us understand whether there is any deep connection between the absence of nonlinear ghost at a certain order in perturbation theory and the corresponding value of the Vainshtein scale.

The main purpose of this note is to show that in the theories considered in $[10,11]$ away from the decoupling limit the nonlinear ghost inevitably arises in the fourth order of 
the perturbative expansion. The Vainshtein scale value becomes therefore unrelated to the absence of ghost if one does not consider the unrealistic decoupling limit of massive gravity.

The inevitable appearance of nonlinear ghost in "Lorentz invariant" massive gravity theories agrees with an independent argument of $[13,14]$ based on helicity decomposition.

Furthermore we argue that the nonlinear ghost can easily be avoided in General Relativity with only three scalar fields, which imitate "Lorentz violating" massive gravity around Minkowski background. This agrees with the results of the papers [15, 16] where Lorentz-violating graviton mass terms have been introduced by hand from the very beginning. Therefore in the theories considered in $[15,16]$ it was not so simple to keep under control such quite exotic phenomena like different maximal velocities for different particle species, superluminal propagation of particles, and violation of the no-hair theorems for the black hole solutions (see $[17,18]$ and references therein). On the other hand our approach allows us to preselect theories as General Relativity with three scalar fields which imitate Lorentz violating gravity but do not lead to dangerous consequences.

\section{Higgs massive gravity}

We employ four scalar fields $\phi^{A}, A=0,1,2,3$, to play the role of Higgs fields. They will acquire a vacuum expectation value proportional to the space-time coordinates $\phi^{A}=\delta_{\beta}^{A} x^{\beta}$ giving mass to the graviton. Let us consider perturbations around Minkowski background,

$$
g^{\mu \nu}=\eta^{\mu \nu}+h^{\mu \nu}, \quad \phi^{A}=x^{A}+\chi^{A}
$$

and define

$$
\begin{aligned}
\bar{h}_{B}^{A} \equiv & \eta_{B C} g^{\mu \nu} \partial_{\mu} \phi^{A} \partial_{\nu} \phi^{C}-\delta_{B}^{A}=h_{B}^{A}+\partial^{A} \chi_{B}+\partial_{B} \chi^{A} \\
& +\partial_{C} \chi^{A} \partial^{C} \chi_{B}+h_{C}^{A} \partial^{C} \chi_{B}+h_{B}^{C} \partial_{C} \chi^{A}+h_{D}^{C} \partial^{D} \chi_{B} \partial_{C} \chi^{A}
\end{aligned}
$$

where indices are moved with the Minkowski metric $\eta_{A B}=(1,-1,-1,-1)$, in particular, $\chi_{B}=\eta_{B C} \chi^{C}$ and $h_{B}^{A}=\eta_{B C} \delta_{\mu}^{A} \delta_{\nu}^{C} h^{\mu \nu}$. After introducing the diffeomorphism invariant variable $\bar{h}_{B}^{A}$ it becomes almost trivial to write the terms that produce massive gravity. In the unitary gauge where $\chi^{A}=0$, we have $\bar{h}_{B}^{A}=h_{B}^{A}=\eta_{B C} \delta_{\mu}^{A} \delta_{\nu}^{C} h^{\mu \nu}$, and hence the FierzPauli term for the graviton mass around broken symmetry background can immediately be obtained from the quadratic term of the following action for the scalar fields

$$
S_{\phi}=\frac{m_{g}^{2}}{8} \int d^{4} x \sqrt{-g}\left[\bar{h}^{2}-\bar{h}_{B}^{A} \bar{h}_{A}^{B}+O\left(\bar{h}^{3}, \ldots\right)\right] .
$$

where by $O\left(\bar{h}^{3}, \ldots\right)$ we denote the terms which are of the third and higher orders in $\bar{h}_{B}^{A}$. In distinction from the Fierz-Pauli action which was introduced by explicit spoiling of the diffeomorphism invariance, our action is manifestly diffeomorphism invariant and only coincides, to leading order, with the Fierz-Pauli action, in the unitary gauge where all perturbations of the scalar fields are set to zero. 


\section{Boulware-Deser nonlinear ghost}

One could, in principle, skip all higher order terms and consider the action

$$
S=-\frac{1}{2} \int d^{4} x \sqrt{-g} R+\frac{m_{g}^{2}}{8} \int d^{4} x \sqrt{-g}\left[\bar{h}^{2}-\bar{h}_{B}^{A} \bar{h}_{A}^{B}\right],
$$

where we set $8 \pi G=1$, as an exact action for massive gravity. The problem then is either the presence of a ghost around the trivial background $\phi^{A}=0$ or the appearance of nonlinear ghost in the broken symmetry phase. To trace the latter one it is convenient to work in some gauge where the scalar field perturbations are not equal to zero. A good choice is the Newtonian gauge in which the metric $g_{\mu \nu}$ takes the form [19]

$$
d s^{2}=(1+2 \phi) d t^{2}+2 S_{i} d t d x^{i}-\left[(1-2 \psi) \delta_{i k}+\tilde{h}_{i k}\right] d x^{i} d x^{k}
$$

where $S_{i, i}=0$ and $\tilde{h}_{i j, i}=\tilde{h}_{i i}=0$. Then the ghost can easily be traced as a dynamical degree of freedom of the scalar field $\chi^{0}$. The field $\chi^{0}$ enters only the $\bar{h}_{0}^{0}$ and $\bar{h}_{0}^{i}$ components, which can be written explicitly as

$$
\bar{h}_{0}^{0}=g^{00}-1+2 g^{00} \dot{\chi}^{0}+g^{00}\left(\dot{\chi}^{0}\right)^{2}+2 g^{0 i} \chi_{, i}^{0}+2 g^{0 i} \dot{\chi}^{0} \chi_{, i}^{0}+g^{i k} \chi_{, i}^{0} \chi_{, k}^{0},
$$

and

$$
\bar{h}_{0}^{i}=g^{0 i}+g^{00} \dot{\chi}^{i}+g^{i k} \chi_{, k}^{0}+\left(g^{0 i}+g^{00} \dot{\chi}^{i}+g^{k 0} \chi_{, k}^{i}\right) \dot{\chi}^{0}+g^{k 0} \chi_{, k}^{i}+g^{l k} \chi_{, l}^{i} \chi_{, k}^{0}+g^{0 k} \chi_{, k}^{0} \dot{\chi}^{i} .
$$

Let us consider only the scalar mode of the massive graviton for which $\chi^{i}=\pi_{, i}$. It was shown in [2] that by using constraints one can express the linear perturbations of the scalar fields in terms of the metric potential $\psi$ as

$$
\begin{gathered}
\pi=\frac{2 \Delta-3 m_{g}^{2}}{m_{g}^{2} \Delta} \psi \\
\chi^{0}=-\frac{2 \Delta+3 m_{g}^{2}}{m_{g}^{2} \Delta} \dot{\psi} .
\end{gathered}
$$

Then the action (3.1) up to second order in perturbations simplifies to

$$
{ }^{(S)} \delta_{2} S=-3 \int d^{4} x\left[\psi\left(\partial_{t}^{2}-\Delta+m_{g}^{2}\right) \psi\right] .
$$

The nonlinear ghost appears in the third order in metric and scalar field perturbations. This is due to the fact that the accidental $U(1)$ symmetry, which makes the scalar field $\chi^{0}$ to be the Lagrange multiplier around Minkowski background, is not preserved on a background slightly deviating from Minkowski space [1]. To prove this it is enough to consider only the third order terms in the action (3.1) which involve the powers of $\dot{\chi}^{0}$. By substituting (3.3) and (3.4) into (3.1) we obtain

$$
\delta_{3} S=\frac{m_{g}^{2}}{2} \int d^{4} x\left\{\left[\left(g^{00}-1+\sqrt{-g}\right) \bar{h}_{i}^{i}+\left(g^{0 i}+\dot{\chi}^{i}-\chi_{, i}^{0}\right)\left(g^{0 i}+\dot{\chi}^{i}\right)\right] \dot{\chi}^{0}+\frac{1}{2} \bar{h}_{i}^{i}\left(\dot{\chi}^{0}\right)^{2}+\ldots\right\},
$$


where by dots we have denoted all other terms not containing time derivatives of $\chi^{0}$. The term, linear in $\dot{\chi}^{0}$, does not induce dynamics for the mode $\chi^{0}$ and simply modifies the constraint equations to second order in perturbations. However, the term proportional to $\left(\dot{\chi}^{0}\right)^{2}$ induces the propagation of $\chi^{0}$ on the background deviating from Minkowski space for which $\bar{h}_{i}^{i} \neq 0$. Thus at nonlinear level there appears an extra scalar degree of freedom which is a ghost. To see this let us express the relevant term in (3.8) entirely in terms of the gravitational potential $\psi$. Taking into account that, to linear order, $\bar{h}_{i}^{i}=6 \psi+2 \Delta \pi$ and using constraint equations (3.5) and (3.6) we find

$$
\delta_{3} S=\frac{m_{g}^{2}}{4} \int d^{4} x\left[\bar{h}_{i}^{i}\left(\dot{\chi}^{0}\right)^{2}+\ldots\right]=\int d^{4} x\left[\Delta \psi\left(\frac{2 \Delta+3 m_{g}^{2}}{m_{g}^{2} \Delta} \ddot{\psi}\right)^{2}+\ldots\right] .
$$

By considering inhomogeneities with $\Delta \psi \gg m_{g}^{2} \psi$ and combining this contribution to the action (3.7) we obtain

$$
\delta S=-3 \int d^{4} x\left[\psi\left(\partial_{t}^{2}-\Delta+m_{g}^{2}\right) \psi-\frac{4}{3 m_{g}^{4}} \Delta \psi(\ddot{\psi})^{2}+\ldots\right] .
$$

Let us assume that there is a background field $\psi_{b}$ and consider small perturbations around this background, that is, $\psi=\psi_{b}+\delta \psi$. Expanding (3.10) to second order in $\delta \psi$ we find that the behavior of linear perturbations is determined by the action

$$
\delta S=-3 \int d^{4} x\left\{\delta \psi\left(\partial_{t}^{2}-\Delta+m_{g}^{2}\right) \delta \psi+\frac{1}{m_{G h}^{2}}\left[\left(\partial_{t}^{2} \delta \psi\right)^{2}+2 \frac{\ddot{\psi}_{b}}{\Delta \psi_{b}}(\Delta \delta \psi)\left(\partial_{t}^{2} \delta \psi\right)\right]+\ldots\right\},
$$

where

$$
m_{G h}^{2}=-\frac{3 m_{g}^{4}}{4 \Delta \psi_{b}}
$$

Let us take for the background field the scalar mode of gravitational wave with the wavenumber $k \sim m_{g}$, for which $\ddot{\psi}_{b} \sim \Delta \psi_{b} \sim m_{g}^{2} \psi_{b}$ and $m_{G h}^{2} \sim m_{g}^{2} / \psi_{b}$. By considering perturbations $\delta \psi$ with wave-numbers $m_{G h}^{2} \gg k^{2} \gg m_{g}^{2}$ and skipping subdominant terms we can rewrite the action above as

$$
\delta S \approx-\frac{3}{m_{G h}^{2}} \int d^{4} x \delta \psi\left(\partial_{t}^{2}+\ldots\right)\left(\partial_{t}^{2}+m_{G h}^{2}+\ldots\right) \delta \psi .
$$

The perturbation propagator is given then by

$$
\frac{1}{\partial^{2}\left(\partial^{2}+m_{G h}^{2}\right)} \simeq \frac{1}{m_{G h}^{2}}\left(\frac{1}{\partial^{2}}-\frac{1}{\partial^{2}+m_{G h}^{2}}\right)
$$

and it obviously describes the scalar mode of the graviton together with non-perturbative Boulware-Deser ghost of mass $m_{G h} \sim m_{g} / \sqrt{\psi_{b}}$. It is clear that when $\psi_{b}$ vanishes the mass $m_{G h}$ becomes infinite and ghost disappears. We have argued in [2] that at energies above Vainshtein scale $\Lambda_{5}=m_{g}^{4 / 5}$ the linearized consideration above breaks down and the scalar fields enter the strong coupling regime. Therefore, if $m_{G h}$ would be larger than $\Lambda_{5}$ then this 
ghost would not be essential. However, in strong enough background $m_{g}<m_{G h}<\Lambda_{5}$ and therefore the nonlinear ghost appears below the Vainshtein scale, where the perturbative expansion is trustable.

Thus, the action (3.1) considered as describing massive gravity has two problems with ghosts: first, there is a linear ghost around the trivial background $\phi^{A}=0$, and second, there is nonlinear ghost around broken symmetry background.

The first ghost is dangerous, because it leads to a strong instability. However, as we have shown in [1], it can be easily avoided by adding to the action (3.1) third and higher order terms in $\bar{h}$. This modification is ambiguous and there is a whole class of theories which reproduce the Fierz-Pauli theory in the lowest order, avoiding linear ghosts around trivial background.

The nonlinear ghost exists only at scales below the Vainshtein energy scale which for the realistic graviton mass is extremely low, about $10^{-20} \mathrm{eV}$. The existence of ghost would allow, for example, a process where ghost-photon pairs are spontaneously produced from the vacuum due to the gravitational interactions [20]. The energies of such photons would be of the order of cutoff scale $\Lambda$. The measurements of the differential photon flux in the diffuse gamma ray emission lead to the bound $\Lambda \leq 3 \mathrm{MeV}$ [21]. Therefore, taking into account that the Vainshtein scale serves as the cutoff scale in Lorentz violating background, where the nonlinear ghost propagates, we conclude that this ghost is completely harmless. Nevertheless, some interesting questions remain. One could inquire whether there is any nonlinear extension of the action (3.1) which is free of the Boulware-Deser ghost and how the absence of the ghost in the corresponding order of a perturbative expansion is related with the concrete value of the Vainshtein scale?

\section{Ghost in nonlinear extensions of massive gravity}

Contrary to $[13,22,23]$, it was claimed recently in $[10,11]$, that there is a unique ghost free nonlinear extension of massive gravity and that this extension is related with $\Lambda_{3}=m_{g}^{2 / 3}$ Vainshtein scale. This claim was proved in $[10,11]$ in the decoupling limit neglecting the vector modes of the graviton. The decoupling limit, while simplifying the calculations, is not physically justified. Therefore, we will determine whether the nonlinear ghost really disappears away from the decoupling limit. The Lagrangian in $[10,11]$ is expressed in terms of the invariants built out of

$$
H_{\mu \nu}=g_{\mu \nu}-\eta_{A B} \partial_{\mu} \phi^{A} \partial_{\nu} \phi^{B}
$$

It is easy to see (as was also noted in [24]) that the invariants built out of $H_{\mu \nu}$, up to sign, coincide with the invariants made of $\bar{h}_{B}^{A}$, in particular,

$$
g^{\mu \nu} H_{\mu \nu}=-\bar{h}, \quad H_{\mu \nu} H^{\mu \nu}=\bar{h}_{B}^{A} \bar{h}_{A}^{B}, \quad \ldots
$$


Let us consider the action $[10,11]$ :

$$
\begin{aligned}
S_{\phi}= & \frac{m_{g}^{2}}{8} \int d^{4} x \sqrt{-g}\left[\bar{h}^{2}-\bar{h}_{A B}^{2}+\frac{1}{2}\left(\bar{h}_{A B}^{3}-\bar{h} \bar{h}_{A B}^{2}\right)-\frac{5}{16} \bar{h}_{A B}^{4}+\frac{1}{4} \bar{h}_{A B}^{3}+\frac{1}{16}\left(\bar{h}_{A B}^{2}\right)^{2}\right. \\
& +c_{3}\left(2 \bar{h}_{A B}^{3}-3 \bar{h} \bar{h}_{A B}^{2}+\bar{h}^{3}+\frac{3}{4}\left(2 \bar{h}_{A B}^{3} \bar{h}-2 \bar{h}_{A B}^{4}+\left(\bar{h}_{A B}^{2}\right)^{2}-\bar{h}_{A B}^{2} \bar{h}^{2}\right)\right) \\
& \left.+d_{5}\left(6 \bar{h}_{A B}^{4}-8 \bar{h}_{A B}^{3} \bar{h}-3\left(\bar{h}_{A B}^{2}\right)^{2}+6 \bar{h}_{A B}^{2} \bar{h}^{2}-\bar{h}^{4}\right)\right]
\end{aligned}
$$

where $c_{3}$ and $d_{5}$ are arbitrary coefficients and we have introduced the shortcut notations

$$
\bar{h}_{A B}^{2}=\bar{h}_{B}^{A} \bar{h}_{A}^{B}, \quad \bar{h}_{A B}^{3}=\bar{h}_{B}^{A} \bar{h}_{C}^{B} \bar{h}_{A}^{C}, \quad \bar{h}_{A B}^{4}=\bar{h}_{B}^{A} \bar{h}_{C}^{B} \bar{h}_{D}^{C} \bar{h}_{A}^{D} .
$$

It was proved $[10,11]$ that this theory is ghost free to fourth order in perturbations in the decoupling limit. The action above corresponds to the Vainshtein scale $\Lambda=m_{g}^{8 / 11}[2]$. Let us investigate whether the ghost really disappears in non-decoupling limit. For this purpose we have to trace all fourth order terms in perturbations which contain time derivatives of $\chi^{0}$. As we have noticed above, the time derivatives of $\chi^{0}$ come only from $\bar{h}_{0}^{0}$ and $\bar{h}_{0}^{i}$ components. Therefore the only terms in (4.3), which survive and could be relevant for a possible ghost are the following

$$
\begin{aligned}
S_{\phi}= & \frac{m_{g}^{2}}{8} \int d^{4} x \sqrt{-g}\left[\left(2 \bar{h}_{0}^{0}-\frac{1}{2}\left(\bar{h}_{0}^{0}\right)^{2}+\frac{1}{4}\left(\bar{h}_{0}^{0}\right)^{3}\right) \bar{h}_{i}^{i}-\frac{1}{4}\left(2 \bar{h}_{0}^{0}-\frac{1}{2}\left(\bar{h}_{0}^{0}\right)^{2}\right) \bar{h}_{i k}^{2}\right. \\
& \left.+2 \bar{h}_{0}^{i} \bar{h}_{0}^{i}-\frac{1}{2} \bar{h}_{0}^{0} \bar{h}_{0}^{i} \bar{h}_{0}^{i}+\frac{1}{4}\left(\bar{h}_{0}^{0}\right)^{2} \bar{h}_{0}^{i} \bar{h}_{0}^{i}+\frac{3}{2} c_{3}\left(2 \bar{h}_{0}^{0}-\frac{1}{2}\left(\bar{h}_{0}^{0}\right)^{2}\right)\left(\left(\bar{h}_{i}^{i}\right)^{2}-\bar{h}_{i k}^{2}\right)+\ldots\right] .
\end{aligned}
$$

We have skipped here the terms which are linear in $\dot{\chi}^{0}$ because they only modify the constraints without inducing the dynamics for $\chi^{0}$. We would like to stress that the particular choice of action (4.3) has lead to nontrivial cancellations of many terms which could have caused the appearance of a ghost. In particular, all contributions which induce the terms proportional to $\left(\dot{\chi}^{0}\right)^{2},\left(\dot{\chi}^{0}\right)^{3},\left(\dot{\chi}^{0}\right)^{4}$ are canceled in the $d_{5}$, term in (4.3). Further nontrivial cancellations happen when we substitute (3.3) and (3.4) in (4.4), and the final result is

$$
\delta_{3} S_{\phi}+\delta_{4} S_{\phi}=\frac{m_{g}^{2}}{8} \int d^{4} x\left[F(\delta g, \chi) \dot{\chi}^{0}+\frac{1}{2}\left(\dot{\chi}^{i}+S_{i}+g^{0 i}+\chi_{, i}^{0}\right)^{2}\left(\dot{\chi}^{0}\right)^{2}+\ldots\right]
$$

where we denote by dots the terms which do not depend on $\dot{\chi}^{0}$. Note that the third and fourth powers of $\dot{\chi}^{0}$ are cancelled. The function $F(\delta g, \chi)$ is some rather long and complicated expression which depends on terms of second and third order in perturbations but does not depend on $\dot{\chi}^{0}$. Because this term does not induce the dynamics of $\chi^{0}$, but simply modifies the constraints, we do not need the explicit form of $F$. Note that the third order terms with second and third powers of $\dot{\chi}^{0}$ are canceled and hence the ghost does not appear in the third order even if we do not consider the decoupling limit. However, in the fourth order in perturbations the nonlinear ghost survives. It is easy to see that this ghost disappears in the decoupling limit in agreement with [10, 11]. In fact, after skipping the 
vector modes, we have $\chi^{i}=\pi_{, i}, S_{i}=0$ and considering the decoupling limit $\left(m_{g}^{2} \rightarrow 0\right)$ we obtain from (3.5) and (3.6) that $\chi^{0} \rightarrow-\dot{\pi}$ and hence the second term in (4.5) vanishes. However, without taking this limit, action (4.5) becomes

$$
\begin{aligned}
\delta_{3} S_{\phi}+\delta_{4} S_{\phi} & =\frac{m_{g}^{2}}{16} \int d^{4} x\left[\left(\dot{\tilde{\chi}}^{i}+S_{i}+\left(\dot{\pi}+\chi^{0}\right)_{, i}\right)^{2}\left(\dot{\chi}^{0}\right)^{2}+\ldots\right] \\
& =\frac{m_{g}^{2}}{16} \int d^{4} x\left[\left(\dot{\tilde{\chi}}^{i}+S_{i}-\frac{6}{\Delta} \dot{\psi}_{, i}\right)^{2}\left(\frac{2 \Delta+3 m_{g}^{2}}{m_{g}^{2} \Delta} \ddot{\psi}\right)^{2}+\ldots\right]
\end{aligned}
$$

where we have taken into account that $\chi^{i}=\pi_{, i}+\tilde{\chi}^{i}$ and $\tilde{\chi}^{i}$ is a vector mode of graviton. Considering small perturbations $\delta \psi$ with wave-numbers $k^{2} \gg m_{g}^{2}$ around some background $\psi_{b}$ and $\tilde{\chi}_{b}^{i}$ we find as in the previous considerations (see (3.10)-(3.12)) that this action describes along with the scalar mode of graviton also a ghost of mass

$$
m_{G h}^{2}=-12 m_{g}^{2}\left(\dot{\tilde{\chi}}_{b}^{i}+S_{i}-\frac{6}{\Delta} \dot{\psi}_{b, i}\right)^{-2}
$$

provided that $m_{G h}^{2}$ satisfies the condition $\partial_{t}^{2} m_{G h}^{-2} \ll 1$. In the background of the scalar gravitational wave $\psi_{b}$ with $k^{2} \simeq m_{g}^{2}$ we have $m_{G h} \sim m_{g} / \psi_{b}$. If the time dependent background fields are strong enough the mass of this ghost is smaller than the Vainshtein scale and can be even as small as the graviton mass. Thus, if one does not consider the decoupling limit of the theory the action (4.3) has a nonlinear ghost in the fourth order of perturbation theory. This ghost cannot be removed by adding fifth and higher order terms and it is inevitable in the theories considered in $[10,11]$.

\section{Can we avoid the nonlinear ghost?}

The theory described by action (4.3) could be a unique candidate for a ghost free massive gravity (to fourth order in perturbations) because it is the only theory which does not have a ghost in the decoupling limit $[10,11]$. It's higher order extension which removes ghost to an arbitrary order is also uniquely determined by the requirement of the absence of ghost in decoupling limit. Thus the theory satisfies the necessary condition to be a ghost free theory. However, this condition is not sufficient to avoid ghost in the full fourth order nonlinear theory. Unfortunately, as we have shown, the theory considered above inevitably has an unremovable nonlinear ghost beginning with the fourth order in perturbations. One can wonder whether there is any way of avoiding this no-go theorem? It is clear that using the variables $H_{\mu \nu}$ defined in (4.1) one is forced to use only the invariants present in (4.3) because otherwise the fundamental diffeomorphism invariance of the theory will be spoiled. On the other hand in our approach the variables $H^{A B}$ are scalars under diffeomorphism transformations. The "internal Lorentz invariance" under scalar fields transformations $\phi^{A} \rightarrow \tilde{\phi}^{A}=\phi^{B} \Lambda_{B}^{A}$ was simply used to imitate massive gravity with Fierz-Pauli term. However, we are not obliged to preserve this fake Lorentz invariance. In fact, there is nothing wrong from the point of view of symmetries to consider for instance the Lagrangian

$$
S_{\phi}=\frac{m_{g}^{2}}{8} \int d^{4} x \sqrt{-g}\left(g^{\mu \nu} \partial_{\mu} \phi^{0} \partial_{\nu} \phi^{0}-1\right)^{2}=\frac{m_{g}^{2}}{8} \int d^{4} x \sqrt{-g}\left(\bar{h}_{0}^{0}\right)^{2}
$$


which is diffeomorphism and Lorentz invariant and simply describes the scalar field $\phi^{0}$ with an unusual kinetic term similar to k-inflation [25, 26]. Therefore, without spoiling any fundamental invariances we could modify the action above by adding to it terms of the form $\left(\bar{h}_{i}^{0}\right)^{2} \bar{h}_{0}^{0},\left(\bar{h}_{i}^{0}\right)^{2}$, etc. It is easy to verify that the only terms in (4.4) responsible for the ghost are

$$
\delta S_{\text {Ghost }} \equiv \frac{m_{g}^{2}}{8} \int d^{4} x\left[2 \bar{h}_{0}^{i} \bar{h}_{0}^{i}-\frac{1}{2} \bar{h}_{0}^{i} \bar{h}_{0}^{i} \bar{h}_{0}^{0}+\frac{1}{4} \bar{h}_{0}^{i} \bar{h}_{0}^{i}\left(\bar{h}_{0}^{0}\right)^{2}\right] .
$$

Therefore subtracting these terms from action (4.3) removes the ghost in the fourth order. In turn this also inevitably modifies the quadratic part of the action and instead of FierzPauli term we obtain

$$
\begin{aligned}
S_{\phi} & =\frac{m_{g}^{2}}{8} \int d^{4} x \sqrt{-g}\left[\bar{h}^{2}-\bar{h}_{B}^{A} \bar{h}_{A}^{B}-2 \bar{h}_{0}^{i} \bar{h}_{0}^{i}+O\left(\bar{h}^{3}, \ldots\right)\right] \\
& =\frac{m_{g}^{2}}{8} \int d^{4} x \sqrt{-g}\left[\left(\bar{h}_{i}^{i}\right)^{2}-\bar{h}_{k}^{i} \bar{h}_{i}^{k}+2 \bar{h}_{0}^{0} \bar{h}_{i}^{i}+O\left(\bar{h}^{3}, \ldots\right)\right] .
\end{aligned}
$$

As a result both scalar and vector modes of the graviton disappear and the action above describes the massive transverse graviton with two degrees of freedom. Note that this result does not contradict Wigner's theorem about the number of degrees of freedom of massive particle with spin-two because in this case the scalar fields background in the broken symmetry phase is not Lorentz invariant. Nevertheless, we would like to stress that in Higgs gravity which produces the massive graviton with two degrees of freedom there is no violation of the fundamental space time Lorentz invariance in distinction from $[15,16]$ where the spacetime Lorentz invariance is violated explicitly by adding distinct mass terms for time and spatial components of the metric perturbations $h_{\mu \nu}$ like $m_{0}^{2} h_{00}^{2}, m_{2}^{2} h_{i j}^{2}, \ldots$ However, effectively the graviton mass term (5.3) could be identified with the theory considered in [15] with $m_{0}^{2}=m_{1}^{2}=0$ and $m_{2}^{2}=m_{3}^{2}=m_{4}^{2}=m_{g}^{2}$. Although this configuration of mass parameters was not conisdered there, the authors would arrive at the same conclusion regarding the number of degrees of freedom of the massive graviton. The effective violation of the spacetime Lorentz invariance in our approach is simply due to the existence of a background scalar field in Minkowski space in a way similar to the violation of this invariance by the cosmic microwave background radiation in our universe. In the case when we had imposed the extra "Lorentz invariance" in the configuration space of the scalar fields we were able to imitate the space-time Lorentz invariance for the graviton mass term simply via redefinition of the scalar fields in the unitary gauge. However, in general when this invariance is absent any scalar fields background violates space-time Lorentz invariance explicitly.

The "Lorentz violating" procedure of removing the nonlinear ghost in Higgs gravity can be extended to any higher orders in the theory considered in $[10,11]$. However, if we allow the "Lorentz violating" terms then there is no need anymore for such extension. We can simply consider

$$
S_{\phi}=\frac{m_{g}^{2}}{8} \int d^{4} x \sqrt{-g}\left[\left(\bar{h}_{i}^{i}\right)^{2}-\bar{h}_{k}^{i} \bar{h}_{i}^{k}\right]
$$

as an exact action of massive gravity on a Lorentz violating background. It is obvious that this action depends only on three scalar fields and does not have any linear and nonlinear 
ghosts around any background. The transverse gravitational degrees of freedom $\tilde{h}_{i k}$ become massive and one could wonder how it will modify the usual Newtonian interaction between massive objects. To answer this question let us consider a static gravitational field produced by a matter for which only $T^{00}$ component of the energy-momentum tensor does not vanish. The metric in this case can be written as

$$
d s^{2}=(1+2 \phi) d t^{2}-(1-2 \psi) \delta_{i k} d x^{i} d x^{k},
$$

and the action for static perturbations derived in [2] (see formulae (28) and (36) there) in the case of (5.4) simplifies to

$$
\begin{aligned}
{ }^{(S)} \delta S= & \int d^{4} x\left\{\psi_{, i} \psi_{, i}+\phi\left[2 \Delta \psi-T^{00}\right]+\frac{m_{g}^{2}}{2}\left[6 \psi^{2}+4 \psi \Delta \pi+\right.\right. \\
& \left.+\left(\Delta \pi \pi_{, i k} \pi_{, i k}-\pi_{, k i} \pi_{, i j} \pi_{, j k}\right)-2 \psi\left(\pi_{, i k} \pi_{, i k}-2(\Delta \pi)^{2}\right)\right] \\
& \left.+O\left(\psi^{3}, \psi^{2} \phi, \psi^{2} \Delta \pi, \phi \psi \Delta \pi \ldots\right)\right\}
\end{aligned}
$$

Varying this action with respect to $\phi, \psi$ and $\pi$, and assuming that $\Delta \pi \ll 1$ we obtain the following equations

$$
\begin{aligned}
\Delta \psi=\frac{T^{00}}{2}, \quad \Delta\left(\psi-\phi-m_{g}^{2} \pi\right)-3 m_{g}^{2} \psi & =0, \\
\Delta \psi+\frac{1}{2}\left(\Delta \pi \pi_{, i k}\right)_{, i k}+\frac{1}{4} \Delta\left(\pi_{, i k} \pi_{, i k}\right)-\frac{3}{4}\left(\pi_{, i j} \pi_{, j k}\right)_{, i k} & =0 .
\end{aligned}
$$

For consistency, we have to include the higher order terms in $\Delta \pi$ because otherwise the first equation in (5.7) would contradict to the equation (5.8). The reason is that the scalar fields in this case are always in strong coupling regime. In particular, given $\psi$ which is induced by the matter source according to Poisson equation and remains unmodified at all, we obtain from (5.8) the following estimate for induced scalar fields

$$
\partial \partial \pi \sim \Delta \pi \sim \sqrt{\psi}
$$

Then considering the spherically symmetric source of mass $M_{0}$ from the second equation in (5.7) one derives

$$
\psi-\phi \simeq O(1) \psi\left(\frac{r}{R_{V}}\right)^{5 / 2} .
$$

At distances much smaller than Vainshtein radius $R_{V}=\left(M_{0} / m_{g}^{4}\right)^{1 / 5}$ we have $\psi=\phi$ with high accuracy and thus we recover General Relativity with corrections which are the same as in the case of Fierz-Pauli mass term (see [2]). However, for $r \gg R_{V}$ the gravitational potential $\phi$ grows as $r^{3 / 2}$, while $\psi$ decays exactly as in Newtonian theory. This is due to the fact that the contribution of the energy of the field $\pi$, induced by the external source of the matter, becomes comparable with the energy of this source at the scales larger than the Vainshtein radius. To find a solution in this range we have to solve exactly the complete nonlinear system of equations. However it is obvious that at distances larger than Vainshtein radius we do not reproduce the results of massive gravity with the Fierz-Pauli 
mass term (see [2]). For the realistic graviton mass, Vainshtein radius for the Sun is huge and before we cross it the contribution of the other mass sources in the universe become important. Smearing the matter distribution and considering the homogeneous universe we find that for $m_{g} \simeq H_{0}$, where $H_{0}$ is the present value of the Hubble constant, the Vainshtein radius in this case is of order of horizon scale $H_{0}^{-1}$. Therefore massive gravity with action (5.4) is in agreement with experiment. An interesting question that needs investigation is to determine how General Relativity will be modified on the horizon scale (a question which could be relevant for the dark energy problem).

\section{How dangerous are ghosts?}

It is clear that the linear ghost around trivial background with $\phi^{A}=0$ is extremely dangerous because it leads to a catastrophic instability of the vacuum and drastically reduces the lifetimes of the particles. We have shown in [1] how this ghost can be easily avoided. In distinction from it the nonlinear ghost seems to be unavoidable in all Lorentz invariant versions of massive gravity. This nonlinear ghost inevitably arises at latest in the fourth order of perturbation theory on a background which slightly deviates from the Minkowski space. How dangerous is this ghost? There exist different opinions on this subject. The main reason why those who think that it is catastrophic is the integration over the Lorentz boosts in order to insure Lorentz invariant cutoff. Leaving the question of the need to integrate over boosts aside we note however that anyway the nonlinear ghost appears only on the background which deviates from the Minkowski space. In turn this background selects the preferable coordinate system where we have a Lorentz violating cutoff on the energy scale below which the ghost exists. This cutoff is the corresponding Vainshtein energy scale, which is extremely low, of order of $10^{-20} \mathrm{eV}$ for the realistic graviton mass. It is clear that the ghost with such energies is completely harmless from the point of view of agreement with experiments [20]. Therefore we believe that the nonlinear ghost in any theory of massive gravity is irrelevant. In such case one could wonder if we can avoid the requirement that the only possible Lorentz invariant graviton mass term is the Fierz-Pauli one? To answer this question let us consider the theory with the action

$$
S_{\phi}=\frac{m_{g}^{2}}{8} \int d^{4} x \sqrt{-g}\left[\bar{h}^{2}-\bar{h}_{B}^{A} \bar{h}_{A}^{B}+\alpha \bar{h}^{2}+O\left(\bar{h}^{3}, \ldots\right)\right] .
$$

It is easy to see that if $\alpha$ is different from zero then already at quadratic order in the action there appears the term $\alpha\left(\dot{\chi}^{0}\right)^{2}$ which inevitably leads to a dangerous linear ghost. Moreover, for $\alpha \sim O(1)$ the Vainshtein scale disappears in this theory. This can be easily seen if we rewrite equations (31), (39) and (41) from our previous paper [2] taking into account the relevant contributions from $\alpha \bar{h}^{2}$ term in action (6.1)

$$
\begin{aligned}
\Delta(\phi+\psi)+\frac{\alpha}{3 \alpha+2} \Delta(\phi-\psi) & =T^{00}+m_{g}^{2} \times(\ldots), \\
(2 \psi-\phi)+\frac{\alpha}{\alpha+1}(\psi+\Delta \pi)+\partial^{4} \pi^{2} & =0 \\
(1+2 \alpha) \psi+\frac{(3 \alpha+2)(\alpha+1)}{2} m_{g}^{2} \pi+\alpha \Delta \pi+\partial^{4} \pi^{2} & =0 .
\end{aligned}
$$


The nonlinear Vainshtein scale was determined before by the requirement that in equation (6.4) the linear term in $\pi$ is equal to the last non-linear term. However, we now have also an extra linear term in this equation which is always larger than the non-linear term if $\Delta \pi \ll 1$. Hence the non-linear term in this equation is negligible and we always remain in the week coupling regime. By considering the scales for which $k^{2} \gg m_{g}^{2}$ it follows from (6.4) that

$$
\Delta \pi=-\frac{(1+2 \alpha)}{\alpha} \psi
$$

Substituting this expression in (6.3) we find that up to the leading order $\psi=\phi$ and hence as it follows from (6.2) curiously enough General Relativity is restored (at least in the leading approximation) without having problem with vDVZ discontinuity $[4,5]$. Nevertheless the above theory is unacceptable because of the linear ghost which exists at all scales up to the Planckian one.

\section{Conclusions}

We have investigated the problem of the non-linear Boulware-Deser ghost in massive gravity. In particular, we have used the gravity Higgs mechanism to study whether the unique theory proposed in $[10,11]$ remains ghost free away from the decoupling regime. Although we have confirmed the result of $[10,11]$ in decoupling limit, we unfortunately find by explicit calculations that a non-linear unremovable ghost reappears in this theory below Vainshtein energy scale in fourth order of perturbation theory away from the decoupling limit. At the same time, as was shown in $[1,2]$, the theories considered in $[10,11]$, can discretely change the Vainshtein scale within the range $M_{0}^{1 / 3} m_{g}^{-2 / 3}<R_{V}<M_{0}^{1 / 5} m_{g}^{-4 / 5}$. Thus, the claim that massive gravity with Vainshtein scale $M_{0}^{1 / 3} m_{g}^{-2 / 3}$ is ghost free is not confirmed in the full theory and moreover the nonlinear ghost problem does not seem to be directly related to the concrete value of the Vainshtein scale.

Higgs massive gravity $[1,2]$ is equivalent to the formulation in $[10,11]$ provided one preserves the "internal Lorentz invariance" in the space of the scalar field configurations. We have argued that in Higgs gravity, in distinction from $[10,11]$, the ghost can be cancelled because the diffeomorphism invariance of the variables $\bar{h}_{B}^{A}$ allows to add appropriate counterterms to cancel the undesired negative energy mode in the action (5.3). This however can only be done if we abandon the Lorentz invariance in the scalar field configuration space without violating the fundamental space-time Lorentz invariance and diffeomorphism invariance of the action in distinction from the Lorentz-violating actions of massive gravity considered in $[15,16]$. As a result the propagators for the scalar and vector modes of the massive graviton vanish and the action (5.3) describes a massive graviton with two physical degrees of freedom.

To summarize, we have shown that even for the simplest action, which at leading order reproduces the Fierz-Pauli mass term and ignoring the higher order terms in $\bar{h}_{B}^{A}$, the Boulware-Deser ghost will arise in third order of perturbation theory. Moving away from the decoupling limit, while keeping the contributions of the vector modes in the action, we have established the existence of the ghost state. We calculated the mass of the ghost mode 
$m_{G h}$ in the short wavelength approximation for perturbations around some locally Lorentz violating background. Moreover, with strong enough background fields it is possible to make the negative energy mode as light as needed within the interval $m_{g}<m_{G h}<\Lambda_{5}$. However, as was argued in [2], above the Vainshtein energy scale $\Lambda_{5}$ the scalar metric perturbations $\psi$ as well as the scalar field perturbations $\chi^{A}$ are in the strong coupling regime and possess no propagator. Therefore, the ghost is propagating on the locally nontrivial background only below the Vainshtein energy scale which for a graviton mass of the order of the present Hubble scale is extremely low and hence the ghost is harmless.

Further, we have shown that by adding terms of higher order in $\bar{h}_{B}^{A}$ to the action with the choice of coefficients corresponding to the Vainshtein scale $\Lambda=m_{g}^{8 / 11}$ the nonlinear ghost disappears from the third order of perturbations. However, away from the decoupling limit the Boulware-Deser ghost, although harmless, appears at the fourth order of perturbation theory and cannot be removed by adding higher order terms to the Lagrangian. This allows us to conclude that the value of the Vainshtein scale which tells us up to which energy scale a perturbation theory of a given order is trustable and the presence of the nonlinear ghost in the theory are two separate issues which do not have to be correlated.

\section{Acknowledgments}

We are grateful to G. Dvali, G. Gabadadze, L. Berezhiani and A. Gruzinov for discussions. The work of AHC is supported in part by the National Science Foundation grant 0854779. L.A and V.M. are supported by TRR 33 "The Dark Universe" and the Cluster of Excellence EXC 153 "Origin and Structure of the Universe".

Open Access. This article is distributed under the terms of the Creative Commons Attribution Noncommercial License which permits any noncommercial use, distribution, and reproduction in any medium, provided the original author(s) and source are credited.

\section{References}

[1] A.H. Chamseddine and V. Mukhanov, Higgs for Graviton: Simple and Elegant Solution, JHEP 08 (2010) 011 [arXiv:1002.3877] [SPIRES].

[2] L. Alberte, A.H. Chamseddine and V. Mukhanov, Massive Gravity: Resolving the Puzzles, JHEP 12 (2010) 023 [arXiv: 1008.5132] [SPIRES].

[3] A.I. Vainshtein, To the problem of nonvanishing gravitation mass, Phys. Lett. B 39 (1972) 393 [SPIRES].

[4] H. van Dam and M.J.G. Veltman, Massive and massless Yang-Mills and gravitational fields, Nucl. Phys. B 22 (1970) 397 [SPIRES].

[5] V.I. Zakharov, Linearized gravitation theory and the graviton mass, JETP Lett. 12 (1970) 312 [SPIRES].

[6] E. Babichev, C. Deffayet and R. Ziour, The Vainshtein mechanism in the Decoupling Limit of massive gravity, JHEP 05 (2009) 098 [arXiv: 0901.0393] [SPIRES]. 
[7] E. Babichev, C. Deffayet and R. Ziour, Recovering General Relativity from massive gravity, Phys. Rev. Lett. 103 (2009) 201102 [arXiv:0907.4103] [SPIRES].

[8] E. Babichev, C. Deffayet and R. Ziour, The recovery of General Relativity in massive gravity via the Vainshtein mechanism, Phys. Rev. D 82 (2010) 104008 [arXiv:1007.4506] [SPIRES].

[9] M. Fierz and W. Pauli, On relativistic wave equations for particles of arbitrary spin in an electromagnetic field, Proc. Roy. Soc. Lond. A 173 (1939) 211 [SPIRES].

[10] C. de Rham and G. Gabadadze, Selftuned Massive Spin-2, Phys. Lett. B 693 (2010) 334 [arXiv: 1006.4367] [SPIRES].

[11] C. de Rham and G. Gabadadze, Generalization of the Fierz-Pauli action, Phys. Rev. D 82 (2010) 4 [arXiv:1007.0443] [SPIRES].

[12] D.G. Boulware and S. Deser, Can gravitation have a finite range?, Phys. Rev. D 6 (1972) 3368 [SPIRES].

[13] G. Dvali, S. Hofmann and J. Khoury, Degravitation of the cosmological constant and graviton width, Phys. Rev. D 76 (2007) 084006 [hep-th/0703027] [SPIRES].

[14] G. Dvali, private communication, to be published.

[15] V.A. Rubakov, Lorentz-violating graviton masses: Getting around ghosts, low strong coupling scale and VDVZ discontinuity, hep-th/0407104 [SPIRES].

[16] S.L. Dubovsky, Phases of massive gravity, JHEP 10 (2004) 076 [hep-th/0409124] [SPIRES].

[17] S.R. Coleman and S.L. Glashow, High-Energy Tests of Lorentz Invariance, Phys. Rev. D 59 (1999) 116008 [hep-ph/9812418] [SPIRES].

[18] S. Dubovsky, P. Tinyakov and M. Zaldarriaga, Bumpy black holes from spontaneous Lorentz violation, JHEP 11 (2007) 083 [arXiv:0706.0288] [SPIRES].

[19] V. Mukhanov, Physical Foundations of Cosmology, Cambridge University Press, Cambridge U.K. (2005).

[20] J.M. Cline, S. Jeon and G.D. Moore, The phantom menaced: Constraints on low-energy effective ghosts, Phys. Rev. D 70 (2004) 043543 [hep-ph/0311312] [SPIRES].

[21] EGRET collaboration, P. Sreekumar et al., EGRET observations of the extragalactic gamma ray emission, Astrophys. J. 494 (1998) 523 [astro-ph/9709257] [SPIRES].

[22] P. Creminelli, A. Nicolis, M. Papucci and E. Trincherini, Ghosts in massive gravity, JHEP 09 (2005) 003 [hep-th/0505147] [SPIRES].

[23] C. Deffayet, G.R. Dvali, G. Gabadadze and A.I. Vainshtein, Nonperturbative continuity in graviton mass versus perturbative discontinuity, Phys. Rev. D 65 (2002) 044026 [hep-th/0106001] [SPIRES].

[24] L. Berezhiani and M. Mirbabayi, Unitarity Check in Gravitational Higgs Mechanism, Phys. Rev. D 83 (2011) 067701 [arXiv:1010.3288] [SPIRES].

[25] C. Armendariz-Picon, T. Damour and V.F. Mukhanov, k-Inflation, Phys. Lett. B 458 (1999) 209 [hep-th/9904075] [SPIRES].

[26] N. Arkani-Hamed, H.-C. Cheng, M.A. Luty and S. Mukohyama, Ghost condensation and a consistent infrared modification of gravity, JHEP 05 (2004) 074 [hep-th/0312099] [SPIRES]. 